\title{
Student Motivation in Computer Networking Courses
}

\author{
Wen-Jung Hsin \\ Associate Professor \\ Information and Computer Science \\ Park University
}

This paper introduces several hands-on projects that have been used to motivate students in learning various computer networking concepts. These projects are shown to be very useful and applicable to the learners' daily tasks and activities such as emailing, Web browsing, and online shopping and banking, and lead to an unexpected byproduct, self-motivation.

\section{I ntroduction}

Teaching theory with hands-on components is shown to be an effective way of conveying information to learners [1]. This is particularly true for teaching computer networking courses, since the use of communication applications such as email and Web browsers has become common for most in recent years. For teaching various computer networking courses, the author has several hands-on projects utilizing advanced computer technologies and freely available software. For example, Java applets can be used for live animation, Ethereal for monitoring packets in transmission, ZoneAlarm for creating personal firewalls, and Microsoft Baseline Security Analyzer for analyzing the security settings of a computer. These technologies and software are evidence that a picture is indeed worth a thousand words. As it turns out, all of these hands-on projects are very useful and applicable to the learners' daily tasks and activities, and lead to a higher form of student motivation, namely, self-motivation.

In each of the following sections, the technology and student motivation associated

As it turns out, all of these hands-on projects are very useful and applicable to the learners' daily tasks and activities, and lead to a higher form of student motivation, namely, selfmotivation. with each assigned project are described in detail. Thus, section 1 deals with Java applets, section 2 with Ethereal, and section 3 with computer network security software.

\section{Section 1: Java applet}

A Java applet is a computer program written in the Java programming language, and can be executed in a client Web browser. For supplemental instruction with their computer networking textbook, Kurose and Ross [2] provide a set of publicly available Java applet animations [3]. These animations, including wireless communications, Hypertext Transfer Protocols (HTTP) and Domain Name System (DNS) protocols, and packet vs. message switching, illustrate how various computer networking applications and protocols work. These applets make networking theory more comprehensible as students are able to explore various networking scenarios by seeing the effect of changing to different parameters and settings.

\section{J ava applet Projects}

During the past three years, the author has observed that computer networking students have benefited from using Java applet animations [3]. To contribute to the pool of available Java applet animations and give back to the computer networking community, beginning in fall 2005, the author has assigned a project in the computer networking theory course (CS365), in which students are to 
write a Java applet to illustrate a networking concept or a computer program to solve a networking problem. The emphasis of the project is on providing an interactive animation to accompany a networking concept or problem and enhance understanding of the subject matter.

\section{Student Motivation with J ava applet}

The main force driving student motivation in this assignment is seeing that their work can benefit other students, potentially worldwide, just as they themselves benefit from using the collection of available applets. Thus far, several such animations have been produced by CS365 students.

In fall 2005, student Joshua McKinzie wrote an animation to illustrate "Selective Repeat" protocol in the network transport layer [4]. His animation has since been published in Kurose and Ross's free Java applet collection [3], in which the authors describe McKinzie's Java applet as a very cool applet animating the Selective Repeat Protocol [3]. "I believe students from all over the world will find [McKinzie's] Selective-Repeat Applet insightful," said Ross [3].

In the same semester, student Nick Kreeger wrote an animation called "Traverse Ping" [5] that reduced excessive error messages for a Nagios [6], a network tool for monitoring host information.

In fall 2006, students had an opportunity to use McKinzie's animation published in Kurose and Ross [3], in what has become an effective method of motivating students to write animations that benefit the computer networking community. Of special note is student Aris Czamanske, who in December 2006 completed an IPV4 calculator that can identify various parts of an IPV4 address [7]. His applet has been used by students majoring in computer networking at Park University, and will be published on the Information and Computer Science Department's website at Park University.

\section{Summary of the Project using J ava applet}

What truly motivates the students in this project is the knowledge that, just as they themselves benefit from using the Java applet animations, their own work can benefit other students, potentially worldwide.

\section{Section 2: Ethereal}

Ethereal is software that can capture and analyze network traffic. The author has used Ethereal in teaching both the theoretical and the hands-on computer networking courses in the past six years at the University of MissouriKansas City and Park University. The author firmly believes that Ethereal has helped students understand various networking concepts, including the protocols and protocol layers, and how systems communicate with each other.

\section{Ethereal Live Demonstration}

Ethereal is a highly effective tool for live demonstrations. The author has used it in class to capture live traffic between a Web browser and Web servers at various popular Web sites such Park University, Yahoo.com, and CNN.com. The captured traffic shows how a request is satisfied between a Web browser and a Web server, and how various protocols, such as DNS, Address Resolution Protocol (ARP), Internet Protocol (IP), Transmission Control Protocol (TCP), and HTTP, work together to retrieve a simple Web page. This frequently opens students' eyes to the complexity of satisfying a simple Web request.

InSight: A Collection of Faculty Scholarship 


\section{Ethereal Projects}

In various networking courses (CS365 - Computer Networking, CS367 Network and Security Administration, and CS373 - Computer Network Security), the author has students perform different projects using Ethereal that include: (1) discovering how various protocols work; (2) examining the differences in the uses of various networking devices such as hub, switch, and router; (3) debugging problems in the communication network; (4) finding out how traffic is re-routed because of a problem in the network; and (5) identifying communication and security loopholes.

\section{Student Motivation in Using Ethereal}

The primary motivation for students in the projects using Ethereal is in the applicability and relevance of the experiment to the students' daily tasks and activities, (i.e., Web browsing, emailing, online shopping or banking, etc.). Students, The primary motivation for as clients, see in detail how their students in the projects communication requests are composed and using Ethereal is in the transmitted to servers, and how the servers in applicability and relevance turn fulfill these requests.

One particular project that increases student knowledge and understanding is the capture and observation of their own of the experiment to the students' daily tasks and activities.

passwords in clear text using File Transfer Protocol (FTP) and TELetype Network protocol (Telnet) [8]. This project proves the importance of using secure protocols such as secure FTP (SFTP) and Secure Shell (SSH) protocols where encryption is used.

In the spring 2007 Computer Network Security (CS373) class, student John Nickell was curious as to how well his ISP, a cable service provider, handled the traffic between itself and his home computer. He used Ethereal, the tool that we taught in class, and found that his ISP uses Post Office Protocol 3 (POP3) for email retrieval and that his password is sent in clear text between his home computer and the ISP. This is especially detrimental in the cable environment, where the flow of information in the cable is shared among its users. Nickell has contacted his ISP, showing what he found, and it is hoped that the ISP can provide a more secure communication channel for its clients. Additionally, Nickell presented his finding as a guest speaker at the Enhancing Teaching and Learning Conference 2007 [9], in which the attendees are mostly educators within Kansas City area. His presentation was well received and added to the knowledge and understanding on the part of many educators at the conference.

\section{Summary of the Project using Ethereal}

Ethereal is a useful software tool that helps uncover the mysteries of networking, by allowing students to see the actual networking in action. A student in my CS373 class motivated himself in using Ethereal to uncover a security problem in the environment provided by his ISP, and presented his finding at an educational conference.

\section{Section 3: Computer Network Security Related Projects}

In spring 2007, Park University offered for the first time Computer Network Security (CS373), a course that introduces students to various security concepts, attacks, vulnerabilities, and countermeasures in computer, network, and information security. Using [10] as a textbook, the course also introduces students to theoretical concepts with many of these put into practice through hands-on projects. 


\section{Computer Network Security Projects}

Each week, in addition to other assignments, four to five hands-on projects are available for the author to give to students, all of whom have a computer of their own, to perform at home.

Depending on the content for a particular week, the projects have varying levels of complexity. Some projects involve substantial setup effort; these include Pretty Good Privacy (PGP) to encrypt e-mails and authenticate the communicating parties, ZoneAlarm to create a personal firewall, Microsoft Baseline Security Analyzer to analyze computer system security, and ShieldsUP by Giberson Research Corporation to examine open ports in the system; whereas other projects are less time-consuming, including those that involve changing the security setting of a Web browser, and changing the setting of a wireless access point to restrict access to the intended users.

\section{Student Motivation in the Security Projects}

The main source of student motivation in the computer network security projects is to be found in the fact that most of the experiments are close to students' hearts. Given the immense number of current computer and network security attacks, students see a need to strengthen the security of computers, their own in particular.

The astonishing fact is that a majority of the students in this class would perform almost every available project every week if their equipment allowed, even though only one per week is required. It is wonderfully rewarding to listen to their bi-weekly presentations on their project experiences. This has been by far one of the most motivated classes in the author's experience at Park University.

\section{Summary of the Projects in Computer Network Security course}

The hands-on projects in the computer network security course are very useful to the students, as they see the applicability to their own computers. Additionally, the need to strengthen computer networks has never been greater, amid the attention to security at the national and international levels in recent years. Students in the class motivated themselves to want to learn more, thereby performing more projects than were required of them.

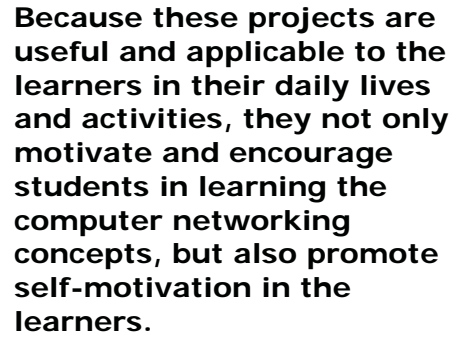
concepts, but also promote self-motivation in the learners.

This paper discussed severa hands-on computer networking projects using various technologies to help students understand and grasp computer networking concepts. Because these projects are useful

and applicable to the learners in their daily lives and activities, they not only motivate and encourage students in learning the computer networking concepts, but also promote self-motivation in the learners. 


\section{References}

[1] F. Gutierrez, "Stingray: A handson approach to learning information security," in Proceedings of the Seventh Conference on Information Technology Education. ACM special interest group in Information Technology Education. 2006.

[2] J.F. Kurose and K.W. Ross, Computer Networking: A Top-Down Approach Featuring the Internet, $3^{\text {rd }}$ edition, Addison-Wesley, 2005.

[3] J.F. Kurose and K.W. Ross, Student Resource Java applet web site,

http://wps.aw.com/aw_kurose_netw ork 3

[4] J. McKinzie, "Selective Repeat,"Student project and poster presentation at the Consortium of Computing in Small Colleges, Northwest Missouri State University in Maryville, Missouri, on April 8, 2006. Also a podium presentation in the First Annual Research and Creative Arts Symposium at Park University, Parkville, Missouri, on April, 23, 2006. Published in www.aw-bc.com/kurose_ross site (student $=>$ Applet) for Computer and Networking textbook by J.F. Kurose and K.W. Ross in May, 2006. Both McKinzie and Hsin are acknowledged in Computer Networking, $4^{\text {th }}$ edition, by J.F. Kurose and K.W. Ross.
[5] N. Kreeger, "Traverse Ping", Student project and poster presentation at the Consortium of Computing in Small Colleges, Northwest Missouri State University in Maryville, Missouri, on April 8, 2006. Also a podium presentation in the First Annual Research and Creative Arts Symposium at Park University, Parkville, Missouri on April 23, 2006.

[6] Nagios, An open source network monitoring tool. http://nagios.org/.

[7] A. Czamanske, "A Java applet in IPv4 address Calculation", Student project and poster presentation at Consortium of Computing in Small Colleges, Drury University, Springfield, Missouri, April 14, 2007. Also a poster presentation in the Second Annual Research and Creative Arts Symposium at Park University, Parkville, Missouri on April 18, 2007.

[8] ]. Cigas, Network Administration Faculty Workshop, 2001. NSF funded project.

[9] W.-J. Hsin, "Enhancing the Understanding of Network Concepts by using Ethereal." Enhancing Teaching and Learning Conference at Park University, Parkville, Missouri, February 24, 2007.

[10] M. Ciampa, Security+ Guide to Network Security Fundamentals, $2^{\text {nd }}$ edition. Thomson Course Technology, 2004.

Hsin received her Ph.D. in the interdisciplinary Telecommunications and Computer Science at University of Missouri - Kansas City. She is currently an associate professor in the Department of Information and Computer Science at Park University. Her research interest is in the areas of computer science education, computer networking, and network security. She has published over 25 journal and conference papers. 